\title{
The mysterious high-latitude O-star HD 93521: new results from XMM-Newton observations
}

\author{
Gregor Rauw and Thierry Morel \\ Institut d'Astrophysique \& Géophysique, University of Liège, \\ Allée du 6 Août 17, B-4000, Liège, Belgium \\ email: rauw@astro.ulg.ac.be
}

\begin{abstract}
The O9.5 Vp star HD 93521 is a well known non-radial pulsator located at a high Galactic latitude. The nature (Population I vs. II) of this star has been the subject of controversy for many years. We report on an XMM-Newton observation of the star that sheds new light on its nature.
\end{abstract}

Keywords. stars: early-type, stars: individual (HD 93521), X-rays: stars

\section{Introduction}

The high Galactic latitude O9.5 Vp star $\operatorname{HD} 93521\left(l_{\mathrm{II}}=183.14^{\circ}, b_{\mathrm{II}}=62.15^{\circ}\right)$, is located $1.4 \mathrm{kpc}$ above the Galactic plane, too far away from any known site of recent star formation for any reasonable value of the runaway velocity. Based on this observation and the fact that the star has a rather low wind velocity, Ebbets \& Savage (1982) argued that HD 93521 could instead be a low-mass Population II star. However, Irvine (1989) and Lennon et al. (1991) concluded that this is unlikely and that the star must be a Population I O-star that formed in the Galactic halo. HD 93521 is one of the fastest rotators $\left(v \sin i=390 \mathrm{~km} \mathrm{~s}^{-1}\right)$ known among O-stars and its absorption lines display bi-periodic $\left(\nu_{1}=13.68, \nu_{2}=8.31 \mathrm{~d}^{-1}\right)$ profile variations that are likely due to non-radial pulsations, although an alternative explanation based on the effect of an orbiting (and accreting) compact companion could not be ruled out entirely (see Rauw et al. 2008 and references therein).

\section{New observations}

To help clarify the nature of HD 93521, we have obtained several new observations.

In the optical, we have obtained ELODIE echelle spectra at the $1.93 \mathrm{~m}$ telescope of the Haute-Provence Observatory (OHP). The lines are very broad, as expected for a fast rotator. Nevertheless, we can identify a number of metallic lines, in agreement with the finding of Lennon et al. (1991) who argued that the metal lines are too strong for a Population II low-mass star.

A $40 \mathrm{ksec}$ XMM-Newton X-ray observation of HD 93521 was obtained in November 2009. HD 93521 is clearly detected, along with a few other sources. We have processed the data with SAS version 8.0 and extracted the EPIC spectra of HD 93521. The spectra can be fitted with a two-temperature thermal plasma model with $\mathrm{kT}_{1}=0.29$ and $\mathrm{kT}_{2}=$ $3.6 \mathrm{keV}$ with solar abundances (see Fig. 1) and an interstellar hydrogen column density of $\mathrm{N}_{\mathrm{H}}=1.3 \times 10^{20} \mathrm{~cm}^{-2}$ (Bohlin et al. 1978). A slightly better quality of the fit is obtained with subsolar abundances $(0.1 \times$ solar $)$. The absorption corrected X-ray flux in 


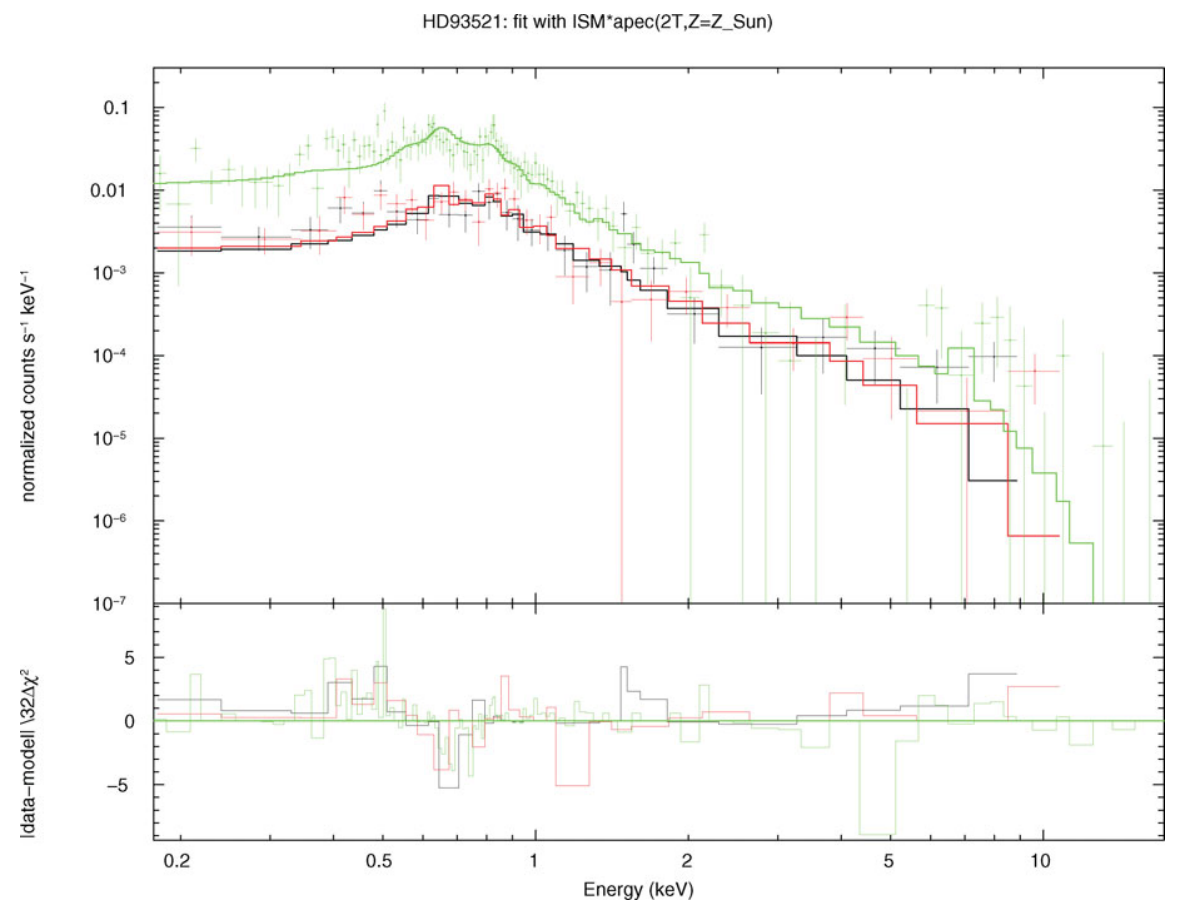

Figure 1. Upper panel: X-ray spectrum of HD 93521 as observed with the three EPIC instruments onboard XMM-Newton. The spectrum was fitted with a two-temperature optically thin thermal plasma model with solar metallicity. Bottom panel : residuals of the fit.

the $0.5-10 \mathrm{keV}$ range $\left(5.75 \times 10^{-14} \mathrm{erg} \mathrm{cm}^{-2} \mathrm{~s}^{-1}\right)$ yields an $\mathrm{L}_{\mathrm{X}} / \mathrm{L}_{\mathrm{bol}}$ ratio (essentially independent of the actual abundances and independent of the distance of the star) of $9.5 \times 10^{-8}$, towards the lower end of the canonical relation for normal O-type stars (Nazé 2009). The presence of a high-temperature emission at $3.6 \mathrm{keV}$ is somewhat unexpected for a wind terminal velocity of $v_{\infty}=400 \mathrm{~km} \mathrm{~s}^{-1}$ (Howarth et al. 1997), although the latter value is probably affected by the fast rotation of the star.

\section{Conclusions}

Our XMM-Newton observations of HD 93521 indicate a rather normal X-ray emission for an O-star, with no obvious signature of a compact companion. However, the X-ray spectra do not allow us to conclude about the metallicity of the star. The optical spectra reveal rather strong, but broad metal lines and we are currently analysing the optical spectrum with a model atmosphere code to derive the chemical composition of the star.

\section{References}

Bohlin, R. C., Savage, B. D., \& Drake, J. F. 1978, ApJ, 224, 132

Ebbets, D. C. \& Savage, B. D. 1982, ApJ, 262, 234

Howarth, I. D., Siebert, K. W., Hussain, G. A. J., \& Prinja, R. K. 1997, MNRAS, 284, 265

Irvine, N. J. 1989, ApJ (Letters), 337, L33

Lennon, D. J., Dufton, P. L., Keenan, F. P., \& Holmgren, D. E. 1991, A\&A, 246, 175

Nazé, Y. 2009, A\&GA, 506, 1055

Rauw, G., De Becker, M., van Winckel, H., Aerts, C. et al. 2008, A\& A, 487, 659 